\title{
Zeitschrift für Didaktik der Naturwissenschaften
}

\author{
Knut Neumann $\cdot$ Stefan Rumann
}

Online publiziert: 2. August 2014

(C) Gesellschaft für Didaktik der Physik und Chemie (GDCP), Fachverband Didaktik der Biologie (FdDB) im VBIO and Springer-Verlag Gmbh Berlin Heidelberg 2014

\section{Liebe Kolleginnen und Kollegen,}

mit dem Jahr 2014 beginnt eine neue Ära in der Entwicklung der Zeitschrift für Didaktik der Naturwissenschaften. In den letzten Jahren haben wir das Editorial regelmäßig mit dem Verweis auf eine überaus positive Entwicklung der Zeitschrift, gemessen u. a. an den steigenden Beitragseinreichungen, begonnen. Eine Entwicklung auf die wir stolz waren - dokumentiert sie doch eine entsprechend positive Entwicklung unserer Disziplin -, die wir aber auch mit Sorge verfolgt haben, weil die zunehmenden Beitragseinreichungen, und damit die steigende Zahl an Verfahren, mit den bestehenden Strukturen nur noch beschränkt zu bewältigen waren. In der Folge kam es häufig zu Verzögerungen im Verfahrensablauf, die für alle Seiten ärgerlich waren.

Bereits früh kam im Herausgeberkreis die Idee der Zusammenarbeit mit einem professionellen Verlag auf. Allerdings verliefen erste Sondierungsgespräche mit verschiedenen Verlagen ernüchternd. Umso mehr freut es uns, dass es uns schließlich mit Unterstützung der Gesellschaft für Didaktik der Chemie und Physik (GDCP), dem Fachverband Didaktik der Biologie (FdDB) im VBIO und nicht zuletzt dem IPN gelungen ist, den national und international im Wissenschaftsbereich überaus renommierten Springer Verlag für die ZfDN zu gewinnen.

Im Spätsommer 2013 hat schließlich die Umstellung begonnen. Das neue Redaktionssystem, das viele von Ihnen bereits kennen, wurde konfiguriert und neue Beiträge sukzessive über das System eingepflegt. Mit den ersten Bei-

K. Neumann $(\triangle) \cdot$ S. Rumann

Leibniz-Insitut für die Pädagogik der Naturwissenschaften und der Mathematik (IPN), Kiel, Deutschland

E-Mail: neumann@ipn.uni-kiel.de trägen im Jahr 2014 wurde schließlich die neue Homepage bei Springer online geschaltet. Dabei erhält jeder neu veröffentlichte Beitrag eine Digital Object Identifier (DOI) Nummer. Damit können Ihre Beiträge ab 2014 weltweit über das Internet als Abstract angezeigt werden. Die bisherigen Jahrgänge sind weiterhin kostenfrei über die Homepage des IPN - oder unter der URL http://archiv.ipn.uni-kiel.de/zfdn/ - verfügbar.

Aber keine Neuerung ohne Probleme - so kam es auch bei der Umstellung zu kleineren Schwierigkeiten, die aber - auch durch Ihre tatkräftige Unterstützung - inzwischen behoben sein sollten. Und trotz der Probleme können wir auch in diesem Editorial auf die weitere positive Entwicklung der Zeitschrift verweisen. Mit über 30 Beitragseinreichungen alleine in der ersten Jahreshälfte haben wir eine neue Bestmarke gesetzt. Zudem konnte für einen Großteil der in diesem Halbjahr eingereichten Beiträge bereits eine erste Herausgeberentscheidung herbeigeführt werden.

Wir haben im Herausgeberkreis diskutiert, wie wir die Erfolge der Zeitschrift, und damit Ihre Erfolge als Autorinnen und Autorinnen bzw. Gutachterinnen und Gutachter, angemessen würdigen können und uns dazu entschieden, das Jahr 2014 mit einem Sonderheft zu beginnen - einem Sonderheft, das die Breite und Qualität der empirischen naturwissenschaftsdidaktischen Forschung widerspiegelt. In diesem Sonderheft mit dem Titel „Naturwissenschaftliche Bildung zwischen Naturwissenschafts- und Fachunterricht" sind vier Beiträge zusammengefasst.

Der Beitrag mit dem Titel „Naturwissenschaftliche Leistungen, demographische Veränderungen und Lehrerbildung" von Olaf Köller repräsentiert die groß angelegten Bildungsvergleichsstudien, deren Befunde häufig den Ausgangspunkt differenzierter naturwissenschaftsdidaktischer Arbeiten bilden. 
Der Beitrag „Fächerübergreifender naturwissenschaftlicher Unterricht: Mythen, Definitionen, Fakten“ von Peter Labudde steht stellvertretend für die vielen guten Beiträge, die den Forschungsstand zu einem Thema nachgehen; hier: der Frage des Naturwissenschaftlichen Unterrichts im Vergleich zu einem Unterricht in den naturwissenschaftlichen Fächern.

Der Beitrag von Dorothee Brovelli mit dem Titel „Integrierte naturwissenschaftliche Lehrerbildung - Entwicklung professioneller Kompetenz bei Lehramtsstudierenden“ schlägt die Brücke vom integrierten naturwissenschaftlichen Unterricht zu einem weiteren hochaktuellen Thema naturwissenschaftsdidaktischer Forschung: der Lehrerbildung.

Und mit dem Beitrag „Vom naturwissenschaftlichen Sachunterricht zum Fachunterricht - Der Übergang von der Grundschule in die weiterführende Schule" von Kornelia Möller schließt sich der Kreis. Er präsentiert die zentralen Ergebnisse eines Forschungsprojekts zur Unterrichtsqualität und deren Determinanten im Übergang vom natur- wissenschaftlichen Sachunterricht zum Unterricht in den naturwissenschaftlichen Fächern.

Wir wünschen Ihnen viel Spaß mit diesem Sonderheft, mit den zahlreichen bereits erschienen Einzelbeiträgen und mit den im zweiten Halbjahr 2014 erscheinenden Beiträge und bedanken uns für Ihre Geduld bei den kleineren und größeren Kinderkrankheiten im Rahmen der Umstellung.

Wir bedanken uns vor allem auch beim Team des Springer-Verlags, das uns in der Umstellung hervorragend betreut hat und stets mit Rat und Tat zur Seite gestanden hat. Wir sind überzeugt mit dem Springer-Verlag einen zuverlässigen Partner gefunden zu haben, mit dem wir die weitere Entwicklung der Zeitschrift erfolgreich leisten werden.

Für den Herausgeberkreis

Knut Neumann

Stefan Rumann 